\title{
Therapeutic Effects of Baoxin Capsules in Combination with Trimetazidine Tablets on Type 2 Diabetes Complicated with Chronic Heart Failure
}

\author{
ANYE DU, WENBIAO ZUO AND PEIYING ZHANG ${ }^{1 * *}$ \\ Department of Endocrinology, Xuzhou City Hospital of TCM, Xuzhou 221009, Jiangsu Province, ${ }^{1}$ Department of Cardiology, \\ Xuzhou Central Hospital, Xuzhou 221009, Jiangsu Province, China
}

Du et al.: Effects of Baoxin Capsules in Combination with Trimetazidine Tablets

To evaluate the therapeutic effects of Baoxin'an capsules combined with trimetazidine tablets on type 2 diabetes with chronic heart failure. A total of 168 patients with type 2 diabetes complicated with chronic heart failure treated in our hospital from January 2016 to December 2017 were selected. They were randomly divided into two groups $(n=84)$, both receiving diet control. On the basis of routine medication, the control group was given trimetazidine tablets. The observation group was administered with Baoxin'an capsules plus trimetazidine tablets. $30 \mathrm{~d}$ were employed as a course of treatment and the entire duration was 
6 mo. The clinical symptoms, cardiac function classifications and the changes of plasma N-terminal pro-Btype natriuretic peptide and high-sensitivity $\mathrm{C}$-reactive protein levels before and after treatment were compared. The overall effective rate of the observation group was $90.48 \%$ which was significantly higher than that of the control group $(73.81 \%)(\chi 2=7.954, p=0.005)$. The increases in blood flow peak velocity ratio of left ventricular diastolic late phase to early phase, left ventricular ejection fraction, cardiac output and stroke volume of the observation group were significantly higher than those of the control group $(p<0.05)$. After treatment, fasting plasma glucose, plasma pro-B-type natriuretic peptide and serum high-sensitivity $C$-reactive protein levels were significantly decreased $(p<0.01)$. The above indices of the observation group were all significantly lower than those of the control group $(p<0.05)$. After treatment, there were no obvious abnormal changes in routine blood, routine urine or liver and kidney function tests. Baoxin'an capsules in combination with trimetazidine tablets can inhibit non-specific inflammatory reaction of type 2 diabetes with chronic heart failure, alleviate myocardial damage and ventricular remodeling, improve cardiac function and enhance clinical therapeutic effects.

Key words: Capsule, trimetazidine, type 2 diabetes, heart failure

Chronic heart failure (CHF) is a common clinical syndrome, as the end stage of various heart diseases. With the improvement of heart disease treatment and population aging, elderly CHF patients account for an increasingly high proportion. The incidence rate of CHF approximately doubles as every $10 \mathrm{y}$ of age increases in the age group of 45 to $94 \mathrm{y}$ old $^{[1]}$.

The common causes for CHF include coronary heart disease, hypertension and rheumatic valvular heart disease. Diabetes is an important risk factor for atherosclerosis and its complications and also an independent risk factor for $\mathrm{CHF}$, which further aggravates the prognosis ${ }^{[2]}$. The risk of CHF in diabetic patients is twice (males) and 5 times (females) that of non-diabetic patients and the rate of CHF complicated with diabetes is up to over $10 \%{ }^{[3]}$, further elevating the mortality and disability rates. The $5 \mathrm{y}$ survival rate of $\mathrm{CHF}$ patients is $46 \%$, but that of CHF complicated with diabetes is only $37 \%$. Besides, the incidence rate of diabetes complicated with CHF in China is soaring recently ${ }^{[4]}$. Therefore, effective therapies are in urgent demand. N-terminal pro-B-type natriuretic peptide (NT-proBNP) reflects not only left ventricular systolic dysfunction, but also left ventricular diastolic dysfunction, which is mainly cleared through the kidneys. After excluding the impact of factors such as renal function, age and weight, NT-proBNP can be used as an independent predictor of prognosis in patients with decompensated heart failure. On the other hand, trimetazidine can improve myocardial energy metabolism, affect the neuroendocrine system, reduce inflammatory cytokines and antioxidative stress and delay ventricular remodeling in CHF patients, thereby

*Address for correspondence

E-mail: anyee4359@163.com improving the cardiac function. In this study, patients with diabetes plus CHF were given Baoxin'an capsules and trimetazidine tablets on the basis of routine medication, aiming to assess the influence on cardiac function and pro-B-type natriuretic peptide (proBNP). This study has been approved by the ethics committee of our hospital and written consent has been obtained from all patients. A total of 168 patients with type 2 diabetes complicated with CHF, who were treated in our hospital from January 2016 to December 2017, were enrolled as subjects. Inclusion criteria: In accordance with the diagnostic criteria for type 2 diabetes and CHF; type 2 diabetes was diagnosed based on the 2011 Criteria for Diagnosis and Treatment of Diabetes of the American Diabetes Association and CHF was diagnosed based on the 2014 Criteria for Diagnosis and Treatment of Heart Failure in China: symptoms of heart failure (e.g. shortness of breath) and/or signs (e.g. edema); left ventricular ejection fraction (LVEF) $\geq 45 \%$; no significant enlargement in the left ventricle and whole heart disclosed by echocardiography and/or chest radiography; evidence of structural cardiac changes such as left atrial enlargement, left ventricular hypertrophy or diastolic heart failure in echocardiogram ${ }^{[5]}$. Patients aged 40-80 y old and New York Heart Association (NYHA) class II-IV; with normal liver and kidney functions. Exclusion criteria: With plasma NT-proBNP level of $<125 \mathrm{pg} / \mathrm{ml}$,

This is an open access article distributed under the terms of the Creative Commons Attribution-NonCommercial-ShareAlike 3.0 License, which allows others to remix, tweak, and build upon the work non-commercially, as long as the author is credited and the new creations are licensed under the identical terms

Accepted 28 December 2020

Revised 24 September 2020

Received 27 June 2020

Indian J Pharm Sci 2020;82(6):1057-1062 
acute heart failure or pump failure, unstable decompensated heart failure (pulmonary edema, hypoperfusion), uncontrollable severe arrhythmia, infective endocarditis, complete atrioventricular block, obstructive cardiomyopathy, pericardial tamponade or pulmonary embolism; complication with various acute and chronic infectious diseases, tumors, blood system diseases, lung, brain, kidney damage or immune system dysfunction; complication with hypertension, hypotension or chronic obstructive emphysema; with history of pulmonary heart disease, stroke or hyperthyroidism; non-compliant patients. The enrolled patients were then randomly divided into two groups $(n=84)$. There were 40 males and 44 females in the control group, who were aged $41-80$ y old, $(58.94 \pm 11.52)$ on average. The duration of CHF was 11 mo to $4.5 \mathrm{y}$, $(40.78 \pm 11.65)$ mo on average. There were 10 cases of NYHA class I, 54 cases of class III and 20 cases of class IV. There were 39 males and 45 females in the observation group, who were aged 41-80 y old, with the mean of $(59.13 \pm 11.79)$. The duration of CHF ranged from 11 mo to $4.5 \mathrm{y},(41.09 \pm 10.98)$ mo on average. There were 11 cases of NYHA class I, 55 cases of class III and 18 cases of class IV. The baseline clinical data of the two groups were not significantly different ( $p>0.05$ ). Both groups received diet control and subcutaneous injection of insulin as part before three meals for hypoglycemic therapy, maintaining the levels of Fasting blood glucose (FBG) and blood glucose $2 \mathrm{~h}$ after meals at $7-8 \mathrm{mmol} / \mathrm{l}$ and $10-11 \mathrm{mmol} / 1$, respectively. According to the 2014 Chinese Guideline for Heart Failure DiagnosisandTreatment, diuretics(hydrochlorothiazide, spironolactone), $\beta$ receptor antagonists, angiotensin converting enzyme inhibitors, aldosterone antagonists and nitrates were used routinely to control blood pressure and blood lipid levels. Meanwhile, low salt diet was kept to regulate electrolyte imbalance. On the basis of the above mentioned mediation, the control group was given trimetazidine tablets (Servier (Tianjin) Pharmaceutical Co., Ltd., China; National Medicine Permit No. H20055465) at $20 \mathrm{mg}, 3$ times/d. The observation group was administered with Baoxin'an capsules (composition: American ginseng, Panax notoginseng and amber powders; Hebei Province
Pharmaceutical Z20060025) on the basis of the control group. The dosage was 3 capsules each time, 3 times/d. $30 \mathrm{~d}$ was employed as a course of treatment and the entire duration was 6 mo. The efficacy of each group was evaluated after one course of treatment. The clinical symptoms, NYHA classification, changes of plasma NT-proBNP and high-sensitivity C-reactive protein (hs-CRP) levels before and after treatment were compared. The NT-proBNP level was measured by the electrochemiluminescence assay using B-type natriuretic peptide (BNP) precursor diagnosis kit (Roche, USA). The hs-CRP level was quantified by immunoturbidimetry with reagent purchased from Shanghai Geng-hygiene Technology Co., Ltd. (China). The cardiac output (CO), stroke volume (SV), LVEF and blood flow peak velocity ratio of left ventricular diastolic late phase to early phase (E/A) were measured by color M-mode Doppler echocardiography before and after treatment, respectively. According to the Diagnostic and Efficacy Criteria for Common Diseases, the treatment outcomes were classified as markedly effective, effective, ineffective and aggravated. Markedly effective: heart failure is basically controlled, or cardiac function is improved by two or more levels; effective: cardiac function is improved by one level but less than two levels; ineffective: cardiac function improved by less than one level; aggravated: cardiac function is exacerbated. Overall effective rate $=$ number of markedly effective and effective cases/total number of cases $\times 100 \%{ }^{[6]}$. All data were analyzed by SPSS 16.0 software. The categorical data were expressed as mean \pm standard deviation and subjected to the $t$ test and analysis of variance. The numerical data were compared by the $\chi^{2}$ test. $\mathrm{p}<0.05$ was considered statistically significant. The overall effective rate of the observation group was $90.48 \%$ which was significantly higher than that of the control group $\left(73.81 \% ; \chi^{2}=7.954, p=0.005\right)$ (Table 1). Before treatment, the two groups had similar $\mathrm{E} / \mathrm{A}, \mathrm{LVEF}, \mathrm{CO}$ and $\mathrm{SV}(\mathrm{p}>0.05)$. The increases in $\mathrm{E} / \mathrm{A}$, LVEF, CO and SV of the observation group were significantly higher than those of the control group $(\mathrm{p}<0.05)$ (Table 2). Before treatment, the two groups had similar fasting plasma glucose (FPG), plasma proBNP and serum hs-CRP levels $(\mathrm{p}>0.05)$. After

TABLE 1: OVERALL EFFECTIVE RATES OF TWO GROUPS

\begin{tabular}{lcccc}
\hline Group & Markedly effective & Effective & Ineffective & Overall effective rate (\%) \\
\hline Observation $(\mathrm{n}=84)$ & 30 & 46 & 8 & 90.48 \\
Control $(\mathrm{n}=84)$ & 20 & 42 & 22 & 73.81 \\
$\mathrm{X}^{2}$ & & & 7.954 \\
$\mathrm{p}$ & & & 0.005 \\
\hline
\end{tabular}


treatment, FPG, plasma proBNP and serum hs-CRP levels were significantly decreased $(p<0.01)$. The above indices of the observation group were all significantly lower than those of the control group $(\mathrm{p}<0.05)$ (Table 3). After treatment, there were no obvious abnormal changes in routine blood, routine urine, or liver and kidney function tests. CHF is characterized by abnormal cardiac function, decreased exercise tolerance and neuroendocrine activation, with the main pathophysiological mechanisms of ventricular remodeling and ventricular structural changes, which reflects cardiac pump dysfunction, i.e. myocardial systolic dysfunction, ventricular filling and/or impaired ejection ability, the stroke volume unable to meet the metabolic needs of the body, insufficient blood perfusion of organs and tissues, long term state of weakness, limited physical activities, fluid retention and breathing difficulty ${ }^{[7]}$. Myocardial systolic dysfunction is caused by primary myocardial damage and long term cardiac capacity and/or stress overload. There are various causes of CHF. In addition to heart and macrovascular diseases, many extracardiac diseases such as infection, diabetes and kidney disease can also cause heart failure, of which type 2 diabetes mellitus is more prominent. Hyperglycemia causes myocardial cell proliferation, hypertrophy and fibrosis by damaging cardiomyocytes through multiple mechanisms such as non-enzymatic glycosylation of proteins, abnormal metabolism of free radicals, abnormal energy metabolism of cardiomyocytes, activation of the renin angiotensin aldosterone system (RAAS) and sympathetic nervous system, so as to lead to abnormal heart contraction and diastolic function. The relationship between $\mathrm{CHF}$ and disorders of glucose metabolism is complex. CHF can cause elevated blood glucose levels. Tension stress in heart failure causes the activation of the neuroendocrine system, the corticosteroids and catecholamines in particular, which not only induces insulin resistance, reduces insulin release of islet receptors and increases hepatic gluconeogenesis, but also aggregates cardiac dysfunction ${ }^{[8]}$. The survey showed that the mortality rate of patients with type 2 diabetes mellitus complicated with CHF was significantly increased. Foreign studies have shown that the annual mortality rate of elderly patients was as high as $32.7 \%{ }^{[9]}$, which was 12 times $(3.2 \%)$ that of patients without diabetes ${ }^{[10]}$. The focus of prevention and treatment of type 2 diabetes mellitus with CHF is to actively control blood glucose and blood pressure to inhibit neurohormone activation, protect cardiomyocyte metabolism and prevent the aggravation of myocardial lesions. In recent years, angiotensin converting enzyme inhibitors (ACEI), $\beta$-blockers, diuretics and digitalis have been proven effective in the treatment of $\mathrm{CHF}$, but due to the combination of diabetes, its treatment has certain limitations. For example, if digitalis is used in patients with coronary heart disease and diabetes, the body's tolerance to drugs is reduced and toxic reactions are likely to occur. Diuretics can significantly improve the symptoms of CHF, but can cause potassium loss in the body, reduce insulin secretion, which is not conducive to the control of blood glucose. Trimetazidine is a novel drug that acts on myocardial metabolism, with the chemical name of 1-(2,3,4-trimethoxybenzyl) piperazine hydrochloride, which can optimize energy metabolism of cardiomyocytes and protect anoxic cardiomyocytes, inhibit oxidative stress and improve heart function. By inhibiting the long chain 3-ketoacylCoA thiolase in mitochondria, it can promote the aerobic metabolism of glucose, improve the utilization of myocardial glucose and meanwhile inhibit the oxidation of "high oxygen-consuming" fatty acids,

TABLE 2: ECHOCARDIOGRAPHIC CHANGES BEFORE AND AFTER TREATMENT

\begin{tabular}{lcccc}
\hline & \multicolumn{2}{c}{ Observation group $(\mathrm{n}=84)$} & \multicolumn{2}{c}{ Control group $(\mathrm{n}=84)$} \\
\hline & Before treatment & After treatment & Before treatment & After treatment \\
E/A & $0.76 \pm 0.24$ & $1.38 \pm 0.32^{*}, \#$ & $0.77 \pm 0.25$ & $1.05 \pm 0.29^{*}$ \\
LVEF $(\%)$ & $37.54 \pm 5.18$ & $49.74 \pm 4.38^{*}, \#$ & $39.08 \pm 5.07$ & $42.98 \pm 4.76^{*}$ \\
CO $(\mathrm{l} / \mathrm{min})$ & $3.21 \pm 0.46$ & $4.62 \pm 0.54^{*}, \#$ & $3.26 \pm 0.48$ & $4.19 \pm 0.45^{*}$ \\
SV $(\mathrm{ml})$ & $53.41 \pm 5.02$ & $64.28 \pm 4.78^{*}, \#$ & $52.38 \pm 4.96$ & $60.54 \pm 5.66^{*}$ \\
\hline
\end{tabular}

Compared with the data before treatment, ${ }^{*} \mathrm{p}<0.05$; compared with the control group, ${ }^{*} \mathrm{p}<0.05$.

TABLE 3: FPG, PLASMA PROBNP AND SERUM Hs-CRP LEVELS BEFORE AND AFTER TREATMENT

\begin{tabular}{lcccc}
\hline & \multicolumn{2}{c}{ Observation group $(\mathrm{n}=84)$} & \multicolumn{2}{c}{ Control group $(\mathrm{n}=84)$} \\
\hline & Before treatment & After treatment & Before treatment & After treatment \\
FPG $(\mathrm{mmol} / \mathrm{l})$ & $10.25 \pm 2.27$ & $7.36 \pm 1.46^{*}, \#$ & $10.26 \pm 2.21$ & $9.21 \pm 2.24^{*}$ \\
ProBNP $(\mathrm{ng} / \mathrm{l})$ & $892.51 \pm 65.95$ & $454.68 \pm 64.42^{*}, \#$ & $894.04 \pm 65.07$ & $648.76 \pm 64.82^{*}$ \\
Hs-CRP $(\mathrm{mg} / \mathrm{l})$ & $6.41 \pm 0.78$ & $1.37 \pm 0.62^{*}, \#$ & $6.40 \pm 0.76$ & $2.65 \pm 0.77^{*}$ \\
\hline
\end{tabular}

Compared with the data before treatment, ${ }^{*} \mathrm{p}<0.05$; compared with the control group, ${ }^{*} \mathrm{p}<0.05$. 
optimize the energy metabolism of cardiomyocytes, so that cardiomyocytes can produce more Adenosine triphosphate (ATP) with limited oxygen and the cardiac systolic function increases; it can also protect the heart by reducing acidosis in cells during myocardial ischemia. In addition, trimetazidine can reduce the release of free radicals and endothelin, thereby improving endothelial function and reducing apoptosis of cardiomyocytes ${ }^{[11,12]}$. This study showed that after the use of trimetazidine in the control group, the cardiac function was significantly improved. The formula of Baoxin'an capsule consists of Panax quinquefolius, Panax notoginseng and amber powders. P. quinquefolius can treat Yin deficiency, benefit Qi, soothe the nerves and contribute to intelligence. In the New Compilation of Materia Medica, P. quinquefolius is evaluated as follows: "tonifying lung, decreasing internal heat, promoting the secretion of saliva or body fluid and removing tiredness, suitable for people with endogenous fire and in poor health". The roots of $P$. quinquefolius contain more than 12 kinds of saponins that have the effects of oxidation resistance, anti-fatigue, anti-stress, anti-myocardial ischemia, anti-myocardial oxidation and increasing myocardial contractility. Besides, $P$. notoginseng can promote blood circulation to remove blood stasis. It contains $P$. notoginseng saponins, flavonoid glycosides, quercetin, etc., with significant anticoagulant effects, which can inhibit platelet aggregation, promote fibrinolysis, reduce whole blood viscosity, increase coronary blood flow, reduce myocardial oxygen consumption, increase cardiac output and have anti-arrhythmic effects. Amber can tranquilize and allay excitement, invigorate the circulation of blood and dissipate blood stasis, with diuretic effects. According to the Supplement of Augmented Materia Medica, amber was used in ancient times to induce urination, remove moisture of spleen, so that spleen could realize the functions of transportation and transformation and Lung Qi was reduced, thereby inducing urination. It has previously been reported that amber mainly contains resin, volatile oil, succinic acid that inhibits the central nervous system and prolongs sleep time $\mathrm{e}^{[13]}$. The incidence and mortality rates of type 2 diabetes mellitus complicated with heart failure are significantly higher than those of non-diabetic patients. Therefore, it is essential for an early detection of cardiac dysfunction in diabetic patients. However, there is no specific clinical manifestation in early $\mathrm{CHF}^{[14]}$. Echocardiography is currently the most useful diagnostic tool for CHF, accurately reflecting the reduction in LVEF and abnormalities in left ventricular structure. Nevertheless, the cardiac systolic and diastolic functions have a certain reserve capacity and the ejection fraction (EF) decline will only occur after the damage reaches a certain level. Therefore, conventional echocardiography cannot easily find cardiac dysfunction in the early stage. Only when the cardiac function is above grade III, the diagnosis of echocardiography is consistent with clinical practice ${ }^{[15]}$. The impact of inflammatory response has been well established in the pathogenesis of CHF. hs-CRP is a marker of inflammatory response and is a non-specific acute phase response protein, which can enhance inflammatory response through the complement system, promote the apoptosis of cardiomyocytes and aggravate myocardial damage. The level of hs-CRP was positively correlated with the severity of CHF, which is an independent predictor of clinical endpoint events and can provide a risk stratification of heart failure ${ }^{[16]}$. Plasma proBNP is a neuroendocrine hormone consisting of 32 amino acid residues, which is mainly derived from the ventricle with diuretic and natriuretic effects. Plasma proBNP can relax blood vessels, inhibit RAAS activity, play an important role in regulating body fluid volume, blood vessel pressure and electrolyte balance and can be elevated in multiple pathophysiological states, especially when CHF occurs. In patients with $\mathrm{CHF}$, hemodynamic changes and neuroendocrine activation can stimulate myocardial cell synthesis, and increase the release of diuretic peptides, which in turn leads to an increase in plasma concentration of diuretic peptides. High amounts of diuretic peptides require more BNP for regulation, so BNP will increase in case of lesions. Hence, the BNP level can reflect the degree of ventricular dysfunction and determine the severity of the disease ${ }^{[17]}$. Plasma BNP concentration is negatively correlated with LVEF and is significantly positively correlated with NYHA cardiac function grade, which can better reflect NYHA grade changes than other indicators $^{[18]}$. Only $83.4 \%$ of $\mathrm{CHF}$ patients can be correctly diagnosed based on BNP $>100 \mathrm{pg} / \mathrm{ml}$. Only by $\mathrm{BNP}<50 \mathrm{pg} / \mathrm{ml}, 96 \%$ of non-CHF patients' plasma can be correctly excluded, suggesting that BNP levels can not only accurately diagnose $\mathrm{CHF}$, but also have a high negative predictive value ${ }^{[19]}$. NT-proBNP is produced together with BNP under the action of protease. Compared with BNP, it has a long half-life, stable blood concentration and the rise of secretion with increased left ventricular load and wall tension. Therefore, CHF can be detected earlier, specifically reflecting the changes of left ventricular function. Using NT-proBNP as the diagnostic and prognostic standard, it has been 
included in the new guidelines for the diagnosis of heart failure and diagnostic criteria of the American College of Cardiology/American Heart Association ${ }^{[20]}$. Regardless, this study still has limitations. The sample size is not large enough. Further in depth studies based on large sample size and multicenter experiences are ongoing in our group. In summary, using traditional Chinese medicine Baoxin'an capsules on the basis of trimetazidine significantly reduced the levels of hsCRP and proBNP compared with those of the control group, accompanied by improved overall effective rate and echocardiographic data. Thus, the combination of Baoxin'an capsule can further inhibit the non-specific inflammatory reaction of patients with type 2 diabetes mellitus and CHF, relieve myocardial damage and ventricular remodeling, improve cardiac function and enhance clinical efficacy.

\section{Conflict of interests:}

The authors declared no conflicts of interest.

\section{REFERENCES}

1. Muntner P, Woodward M, Mann DM, Shimbo D, Michos ED, Blumenthal RS, et al. Comparison of the Framingham Heart Study hypertension model with blood pressure alone in the prediction of risk of hypertension: the Multi-Ethnic Study of Atherosclerosis. Hypertension 2010;55(6):1339-45.

2. McDonnell MG, Young IS, Nicholls DP, Archbold GP, Graham CA. Genetics of the lipoprotein lipase gene and hypertriglyceridaemia. Br J Biomed Sci 2003;60(2):84-8.

3. Varma C, Camm AJ. Pacing for heart failure. Lancet 2001;357(9264):1277-83.

4. Dongfeng $G$, Guangyong $H$, Jiang HE. Investigation of prevalence and distributing feature of chronic heart failure in Chinese adult population. Chin J Cardiol 2003;31(1):3-6.

5. Chinese guidelines for the diagnosis and treatment of heart failure 2014. Zhonghua Xin Xue Guan Bing Za Zhi 2014;42(2):98-122.

6. Wu SZ. Diagnosis and treatment of common diseases (standard). China Press of Traditional Chinese Medicine, Beijing 1999.

7. Tsai MF, Hwang SL, Tsay SL, Wang CL, Tsai FC, Chen CC, et al. Predicting trends in dyspnea and fatigue in heart failure patients' outcomes. Acta Cardiol Sin 2013;29(6):488-95.

8. Benedict CR, Weiner DH, Johnstone DE, Bourassa MG, Ghali $\mathrm{JK}$, Nicklas J, et al. Comparative neurohormonal responses in patients with preserved and impaired left ventricular ejection fraction: Results of the studies of left ventricular dysfunctions (SOLVD) registry. J Am Coll Cardiol 1993;22(4S1):A146-53.

9. Metra M, O'Connor CM, Davison BA, Cleland JG, Ponikowski $\mathrm{P}$, Teerlink JR, et al. Early dyspnoea relief in acute heart failure: prevalence, association with mortality and effect of rolofylline in the PROTECT Study. Eur Heart J 2011;32(12):1519-34.

10. Vaur L, Gueret P, Lievre M, Chabaud S, Passa P. Development of congestive heart failure in type 2 diabetic patients with microalbuminuria or proteinuria: observations from the DIABHYCAR (type 2 DIABetes, Hypertension, Cardiovascular Events and Ramipril) study. Diabetes care 2003;26(3):855-60.

11. Zhang L, Lu Y, Jiang H, Zhang L, Sun A, Zou Y, et al. Additional use of trimetazidine in patients with chronic heart failure: a meta-analysis. J Am Coll Cardiol 2012;59(10):91322.

12. Zhao Y, Peng L, Luo Y, Li S, Zheng Z, Dong R, et al. Trimetazidine improves exercise tolerance in patients with ischemic heart disease. Herz 2016;41(6):514-22.

13. Yang L, Li TT, Chu YT, Chen K, Tian SD, Chen XY, et al. Traditional Chinese medical comprehensive therapy for cancer-related fatigue. Chin J Integr Med 2016;22(1):67-72.

14. Cubbon RM, Adams B, Rajwani A, Mercer BN, Patel PA, Gherardi G, et al. Diabetes mellitus is associated with adverse prognosis in chronic heart failure of ischaemic and nonischaemic aetiology. Diabetes Vasc Dis Res 2013;10(4):330-6.

15. Boonman-de Winter LJ, Rutten FH, Cramer MJ, Landman MJ, Liem AH, Rutten GE, et al. High prevalence of previously unknown heart failure and left ventricular dysfunction in patients with type 2 diabetes. Diabetologia 2012;55(8):215462.

16. Kozdag G, Ertas G, Kilic T, Acar E, Agir A, Sahin T, et al. Elevated level of high-sensitivity C-reactive protein is important in determining prognosis in chronic heart failure. Med Sci Monit 2010;16(3):CR156-61.

17. Mueller T, Gegenhuber A, Poelz W, Haltmayer M. Diagnostic accuracy of $\mathrm{B}$ type natriuretic peptide and amino terminal proBNP in the emergency diagnosis of heart failure. Heart 2005;91(5):606-12.

18. Huang YT, Tseng YT, Chu TW, Chen J, Lai MY, Tang WR, et al. N-terminal pro b-type natriuretic peptide (NT-pro-BNP)based score can predict in-hospital mortality in patients with heart failure. Sci Rep 2016;6(1):1-3.

19. Dieplinger B, Egger M, Leitner I, Firlinger F, Poelz W, Lenz $\mathrm{K}$, et al. Interleukin 6, galectin 3, growth differentiation factor 15 and soluble ST2 for mortality prediction in critically ill patients. J Crit Care 2016;34:38-45.

20. Ong KL, Allison MA, Cheung BM, Wu BJ, Barter PJ, Rye KA. Trends in C-reactive protein levels in US adults from 1999 to 2010. Am J Epidemiol 2013;177(12):1430-42. 